\title{
Larger families of solutions to some Diophantine equations
}

\author{
Lyes Ait-Amrane \\ USTHB, Faculty of Mathematics, LATN Laboratory, \\ BP 32, El Alia, 16111, Bab Ezzouar, Algiers, Algeria \\ Ecole nationale Supérieure d'Informatique (ESI) \\ BP 68M Oued Smar, 16270, El Harrach, Algiers, Algeria \\ e-mail: lyesait@gmail.com, I_ait_amrane@esi.dz
}

Received: 22 July 2018

Revised: 22 January 2019

Accepted: 25 January 2019

\begin{abstract}
In this paper, we give three identities involving the Lucas sequences of the first kind and of the second kind in order to obtain infinite families of solutions to some Diophantine equations. Some of these families are new and the others are larger than those known until now.
\end{abstract}

Keywords: Fibonacci and Lucas numbers, Lucas sequences, Diophantine equations.

2010 Mathematics Subject Classification: 11B37, 11B39, 11D45, 40 C05.

\section{Introduction}

There are many Diophantine equations that have all solutions given in terms of particular Lucas sequences as Fibonacci and Lucas numbers (e.g. [1, 2, 3]). Keskin [4] exhibits families of solutions involving Fibonacci and Lucas numbers to some Diophantine equations. He says that it is a bit difficult and interesting to give all integer solutions to these Diophantine equations. The aim of this paper is to extend the work of Keskin [4] to Lucas sequences in order to obtain larger families of solutions to all Diophantine equations given in [4]. The Lucas sequence of the first kind $\left(F_{n}^{(s, r)}\right)_{n \geq 0}$ is defined by the initial values $F_{0}^{(s, r)}=0$ and $F_{1}^{(s, r)}=1$ with the recurrence relation $F_{n+1}^{(s, r)}=s F_{n}^{(s, r)}+r F_{n-1}^{(s, r)}$ for $n \geq 1$, where $s$ and $r$ are any non-zero integers. Its companion sequence $\left(L_{n}^{(s, r)}\right)_{n \geq 0}$, i.e., the Lucas sequence of the second kind, is defined by the initial 
values $L_{0}^{(s, r)}=2$ and $L_{1}^{(s, r)}=s$ with the same recurrence relation $L_{n+1}^{(s, r)}=s L_{n}^{(s, r)}+r L_{n-1}^{(s, r)}$. The numbers $F_{n}^{(1,1)}$ and $L_{n}^{(1,1)}$ are the classical Fibonacci and Lucas numbers, respectively. Let $\alpha=(s+\sqrt{\Delta}) / 2$ and $\beta=(s-\sqrt{\Delta}) / 2$ be the roots of the polynomial $x^{2}-s x-r$, where $\Delta=s^{2}+4 r$. These roots satisfy

$$
\alpha+\beta=s, \quad \alpha \beta=\frac{1}{4}\left(s^{2}-\Delta\right)=-r \quad \text { and } \quad \alpha-\beta=\sqrt{\Delta} .
$$

Throughout this paper, we will assume that $\alpha \neq \beta$, then we have the well-known Binet's formulas

$$
F_{n}^{(s, r)}=\frac{\alpha^{n}-\beta^{n}}{\sqrt{\Delta}} \quad \text { and } \quad L_{n}^{(s, r)}=\alpha^{n}+\beta^{n}, \quad n \geq 0
$$

From (1) and (2) we get that Lucas sequences for negative subscripts are defined by

$$
F_{-n}^{(s, r)}=-(-r)^{-n} F_{n}^{(s, r)} \quad \text { and } \quad L_{-n}^{(s, r)}=(-r)^{-n} L_{n}^{(s, r)} .
$$

In particular, we have

$$
F_{-n}^{(s, 1)}=(-1)^{n+1} F_{n}^{(s, 1)}, \quad L_{-n}^{(s, 1)}=(-1)^{n} L_{n}^{(s, 1)} .
$$

and

$$
F_{-n}^{(s,-1)}=-F_{n}^{(s,-1)}, \quad L_{-n}^{(s,-1)}=L_{n}^{(s, r)} .
$$

Thus, these particular sequences give also integers for negative subscripts.

In order to alleviate the notations, we will note by $\left(u_{n}\right)$ and $\left(v_{n}\right)$ the sequences $\left(F_{n}^{(s, r)}\right)$ and $\left(L_{n}^{(s, r)}\right)$, respectively, except when we give precise values to $s$ or $r$. There are many identities satisfied by the terms of Lucas sequences that are generalizations of those satisfied by Fibonacci numbers, we list here those needed for our paper.

$$
\begin{gathered}
v_{n}=u_{n+1}+r u_{n-1}, \\
v_{n}^{2}-\Delta u_{n}^{2}=4(-r)^{n}, \\
\lambda u_{n}+r u_{n-1}=\lambda^{n}, \\
v_{n} v_{m}+\Delta u_{n} u_{m}=2 v_{n+m},
\end{gathered}
$$

where $\lambda \in\{\alpha, \beta\}$ and $n, m \in \mathbb{Z}$. For more information about Lucas sequences one can see $[5,6,7]$.

In this paper we give some new identities satisfied by the terms of Lucas sequences, from which we deduce families of solutions to Diophantine equations. Some of these Diophantine equations are presented in [4]. Here we obtain larger families of solutions to these equations than those given in [4]. As an example, the solutions given in [4] to the Diophantine equation $z^{2}+x^{2}+y^{2}=x y z+4$ are $(x, y, z)=\left(L_{2 n}^{(1,1)}, L_{2 m}^{(1,1)}, L_{2 n+2 m}^{(1,1)}\right)$ and $\left(a^{2}-2, a, a\right)$ where $a$ is an integer. In this paper, the solutions we obtain are $(x, y, z)=\left(L_{2 n}^{(s, 1)}, L_{2 m}^{(s, 1)}, L_{2 n+2 m}^{(s, 1)}\right)$ and $(x, y, z)=\left(L_{n}^{(s,-1)}, L_{m}^{(s,-1)}, L_{n+m}^{(s,-1)}\right)$. 
The Diophantine equations presented in [4] are

$$
\begin{gathered}
z^{2}+x^{2}+y^{2}=x y z+4 \\
z^{2}-x^{2}-y^{2}=x y z+4 \\
z^{2}-x^{2}+y^{2}=x y z-4 \\
z^{2}-5 x^{2}-5 y^{2}=5 x y z+4 \\
z^{2}+5 x^{2}+5 y^{2}=5 x y z+4 \\
z^{2}-5 x^{2}+5 y^{2}=5 x y z-4 \\
5 z^{2}-x^{2}+5 y^{2}=5 x y z-4
\end{gathered}
$$

Note that if $(a, b, c)$ is a solution to one of the above equations, then also are $(-a,-b, c)$, $(-a, b,-c)$ and $(a,-b,-c)$. Note also that if $\left(x_{1}, x_{2}, x_{3}\right)$ is a solution of one of almost all the preceding equations, then there exists at least one $\sigma \in S_{3}$ such that $\left(x_{\sigma(1)}, x_{\sigma(2)}, x_{\sigma(3)}\right)$ is also a solution of the same equation, where $S_{3}$ denotes the symmetric group. For example, if $\left(x_{1}, x_{2}, x_{3}\right)$ is a solution of the first equation, then $\left(x_{\sigma(1)}, x_{\sigma(2)}, x_{\sigma(3)}\right)$ is also a solution for any $\sigma \in S_{3}$.

\section{Some new identities}

In this section, we present some new identities involving the Lucas sequences $\left(u_{n}\right)$ and $\left(v_{n}\right)$ which were defined previously. Recall that $r$ and $s$ are the integers defining the recursive relation of the sequences $\left(u_{n}\right)$ and $\left(v_{n}\right)$.

Lemma 1. If $X$ is a square matrix with $X^{2}=s X+r I$, then $X^{n}=u_{n} X+r u_{n-1}$ I for all $n \in \mathbb{Z}$.

Proof. The identity $X^{n}=u_{n} X+r u_{n-1} I$ is trivially checked for $n=0$ and 1 using the fact that $u_{-1}=1 / r$. For $n \geq 2$ the result follows from a straightforward induction. It remains to show that $X^{-n}=u_{-n} X+r u_{-n-1} I$ for $n<0$. Let $Y=s I-X=-r X^{-1}$, it follows that $Y^{2}=s Y+r I$. Which shows that $Y^{n}=u_{n} Y+r u_{n-1} I$. That is

$$
\left(-r X^{-1}\right)^{n}=-u_{n} X+u_{n+1} I .
$$

From which we deduce that

$$
X^{-n}=-(-r)^{-n} u_{n} X+(-r)^{-n} u_{n+1} I
$$

Now, using (3) we get

$$
X^{-n}=u_{-n} X+r u_{-n-1} I .
$$

Proposition 2. Let $A=\left(\begin{array}{ll}\alpha & 0 \\ 1 & \beta\end{array}\right)$, then

$$
A^{n}=\left(\begin{array}{cc}
\alpha^{n} & 0 \\
u_{n} & \beta^{n}
\end{array}\right), \quad n \in \mathbb{Z} .
$$


Proof. Since $A^{2}=s A+r I$, then from Lemma 1 and identity (6) we obtain

$$
A^{n}=u_{n} A+r u_{n-1} I=\left(\begin{array}{cc}
\alpha u_{n}+r u_{n-1} & 0 \\
u_{n} & \beta u_{n}+r u_{n-1}
\end{array}\right)=\left(\begin{array}{cc}
\alpha^{n} & 0 \\
u_{n} & \beta^{n}
\end{array}\right) .
$$

Theorem 3. Let $n$ and $m$ be integers, then

$$
(-1)^{n+m} v_{n+m} v_{n} v_{m}+4 r^{n+m}=(-1)^{n+m} v_{n+m}^{2}+(-1)^{n} r^{m} v_{n}^{2}+(-1)^{m} r^{n} v_{m}^{2}
$$

and

$$
(-1)^{n+m} v_{n+m}^{2}-\Delta r^{m}(-1)^{n} u_{n}^{2}-\Delta r^{n}(-1)^{m} u_{m}^{2}=(-1)^{n+m} \Delta v_{n+m} u_{n} u_{m}+4 r^{n+m} .
$$

Proof. We have

$$
\sqrt{\Delta} \alpha^{n}=(\alpha-\beta) \alpha^{n}=\alpha^{n+1}-\beta \alpha^{n}=\alpha^{n+1}-(\alpha \beta) \alpha^{n-1}=\alpha^{n+1}+r \alpha^{n-1} .
$$

In the same way, we have $\beta^{n+1}+r \beta^{n-1}=-\sqrt{\Delta} \beta^{n}$. Thus, from Proposition 2 and (4) we get

$$
A^{n+1}+r A^{n-1}=\left(\begin{array}{cc}
\sqrt{\Delta} \alpha^{n} & 0 \\
v_{n} & -\sqrt{\Delta} \beta^{n}
\end{array}\right)
$$

from which we obtain

$$
\left(A^{n+1}+r A^{n-1}\right)\left(A^{m+1}+r A^{m-1}\right)=\left(\begin{array}{cc}
\Delta \alpha^{n+m} & 0 \\
\sqrt{\Delta}\left(\alpha^{m} v_{n}-\beta^{n} v_{m}\right) & -\Delta \beta^{n+m}
\end{array}\right) .
$$

On the other hand, we have

$$
\begin{aligned}
\left(A^{n+1}+r A^{n-1}\right)\left(A^{m+1}+r A^{m-1}\right) & =A^{n+m+2}+2 r A^{n+m}+r^{2} A^{n+m-2} \\
& =\left(\begin{array}{ll}
\alpha^{n+m+2}+2 r \alpha^{n+m}+r^{2} \alpha^{n+m-2} & 0 \\
u_{n+m+2}+2 r u_{n+m}+r^{2} u_{n+m-2} & \cdots
\end{array}\right) .
\end{aligned}
$$

We deduce that

$$
\begin{aligned}
\sqrt{\Delta}\left(\alpha^{m} v_{n}-\beta^{n} v_{m}\right) & =u_{n+m+2}+2 r u_{n+m}+r^{2} u_{n+m-2} \\
& =s u_{n+m+1}+3 r u_{n+m}+r u_{n+m}-s r u_{n+m-1} \\
& =s^{2} u_{n+m}+s r u_{n+m-1}+4 r u_{n+m}-s r u_{n+m-1} \\
& =\Delta U_{n+m} .
\end{aligned}
$$

That is, $\sqrt{\Delta} U_{n+m}=\alpha^{m} v_{n}-\beta^{n} v_{m}$. Thus,

$$
\begin{aligned}
\Delta U_{n+m}^{2} & =\left(\alpha^{m} v_{n}-\beta^{n} v_{m}\right)\left(\alpha^{n} v_{m}-\beta^{m} v_{n}\right) \\
& =\alpha^{n+m} v_{n} v_{m}-(\alpha \beta)^{m} v_{n}^{2}-(\alpha \beta)^{n} v_{m}^{2}+\beta^{n+m} v_{n} v_{m} \\
& =v_{n+m} v_{n} v_{m}-(-r)^{m} v_{n}^{2}-(-r)^{n} v_{m}^{2}
\end{aligned}
$$


Using identity (5), we obtain

$$
v_{n+m} v_{n} v_{m}-(-r)^{m} v_{n}^{2}-(-r)^{n} v_{m}^{2}=v_{n+m}^{2}-4(-r)^{n+m},
$$

i.e.,

$$
(-1)^{n+m} v_{n+m} v_{n} v_{m}+4 r^{n+m}=(-1)^{n+m} v_{n+m}^{2}+(-1)^{n} r^{m} v_{n}^{2}+(-1)^{m} r^{n} v_{m}^{2} .
$$

This proves (8). Now replacing $v_{n}^{2}=\Delta u_{n}^{2}+4(-r)^{n}$ and $v_{m}^{2}=\Delta u_{m}^{2}+4(-r)^{m}$ in (5) we get

$$
(-1)^{n+m} v_{n+m} v_{n} v_{m}=(-1)^{n+m} v_{n+m}^{2}+(-1)^{n} r^{m} \Delta u_{n}^{2}+(-1)^{m} r^{n} \Delta u_{m}^{2}+4 r^{n+m} .
$$

Using (7), we obtain

$$
(-1)^{n+m} v_{n+m}\left(2 v_{n+m}-\Delta u_{n} u_{m}\right)=(-1)^{n+m} v_{n+m}^{2}+(-1)^{n} r^{m} \Delta u_{n}^{2}+(-1)^{m} r^{n} \Delta u_{m}^{2}+4 r^{n+m},
$$

i.e.,

$$
(-1)^{n+m} v_{n+m}^{2}-\Delta r^{m}(-1)^{n} u_{n}^{2}-\Delta r^{n}(-1)^{m} u_{m}^{2}=(-1)^{n+m} \Delta v_{n+m} u_{n} u_{m}+4 r^{n+m} .
$$

This proves (9).

Theorem 4. Let $n$ and $m$ be integers, then

$$
(-1)^{n+m} \Delta u_{n+m}^{2}-(-1)^{n} r^{m} v_{n}^{2}+\Delta(-1)^{m} r^{n} u_{m}^{2}=\Delta(-1)^{n+m} u_{n+m} v_{n} u_{m}-4 r^{n+m} .
$$

Proof. We just saw that

$$
A^{n+1}+r A^{n-1}=\left(\begin{array}{cc}
\sqrt{\Delta} \alpha^{n} & 0 \\
v_{n} & -\sqrt{\Delta} \beta^{n}
\end{array}\right) .
$$

Thus, by calculating $\left(A^{n+1}+r A^{n-1}\right) A^{m}$ and $A^{m}\left(A^{n+1}+r A^{n-1}\right)$ in two different ways, it can be shown that

$$
v_{n+m}=\alpha^{m} v_{n}-\sqrt{\Delta} \beta^{n} u_{m} \quad \text { and } \quad v_{n+m}=\sqrt{\Delta} \alpha^{n} u_{m}+\beta^{m} v_{n} .
$$

We deduce that

$$
\begin{aligned}
v_{n+m}^{2} & =\left(\alpha^{m} v_{n}-\sqrt{\Delta} \beta^{n} u_{m}\right)\left(\sqrt{\Delta} \alpha^{n} u_{m}+\beta^{m} v_{n}\right) \\
& =\sqrt{\Delta}\left(\alpha^{n+m}-\beta^{n+m}\right) v_{n} u_{m}+(-1)^{m} r^{m} v_{n}^{2}-\Delta(-1)^{n} r^{n} u_{m}^{2} \\
& =\Delta u_{n+m} v_{n} u_{m}+(-1)^{m} r^{m} v_{n}^{2}-\Delta(-1)^{n} r^{n} u_{m}^{2}
\end{aligned}
$$

Using (5) we get

$$
\Delta u_{n+m}^{2}+4(-1)^{n+m} r^{n+m}=\Delta u_{n+m} v_{n} u_{m}+(-1)^{m} r^{m} v_{n}^{2}-\Delta(-1)^{n} r^{n} u_{m}^{2},
$$

i.e.,

$$
(-1)^{n+m} \Delta u_{n+m}^{2}-(-1)^{n} r^{m} v_{n}^{2}+\Delta(-1)^{m} r^{n} u_{m}^{2}=\Delta(-1)^{n+m} u_{n+m} v_{n} u_{m}-4 r^{n+m} .
$$




\section{Families of solutions to some Diophantine equations}

Theorems 3 and 4 allow us to state the following results, in which we find the equations described in [4] with larger families of solutions.

Corollary 5. Let $n$ and $m$ be integers, then $(x, y, z)=\left(L_{2 n}^{(s, 1)}, L_{2 m}^{(s, 1)}, L_{2 n+2 m}^{(s, 1)}\right)$ and $(x, y, z)=$ $\left(L_{n}^{(s,-1)}, L_{m}^{(s,-1)}, L_{n+m}^{(s,-1)}\right)$ are solutions of the equation $x^{2}+y^{2}+z^{2}=x y z+4$. Moreover, $(x, y, z)=\left(L_{2 n+1}^{(s, 1)}, L_{2 m+1}^{(s, 1)}, L_{2 n+2 m+2}^{(s, 1)}\right)$ is a solution of the equation $z^{2}-x^{2}-y^{2}=x y z+4$ and $(x, y, z)=\left(L_{2 n}^{(s, 1)}, L_{2 m+1}^{(s, 1)}, L_{2 n+2 m+1}^{(s, 1)}\right)$ is a solution of $z^{2}-x^{2}+y^{2}=x y z+4$.

Corollary 6. Let $n$ and $m$ be integers, then $(x, y, z)=\left(F_{2 n}^{(s, 1)}, F_{2 m}^{(s, 1)}, L_{2 n+2 m}^{(s, 1)}\right)$ and $(x, y, z)=$ $\left(F_{n}^{(s,-1)}, F_{m}^{(s,-1)}, L_{n+m}^{(s,-1)}\right)$ are solutions of the equation $z^{2}-\Delta x^{2}-\Delta y^{2}=\Delta x y z+4$. Moreover, $(x, y, z)=\left(F_{2 n+1}^{(s, 1)}, F_{2 m+1}^{(s, 1)}, L_{2 n+2 m+2}^{(s, 1)}\right)$ is a solution of the equation $z^{2}+\Delta x^{2}+\Delta y^{2}=\Delta x y z+4$ and $(x, y, z)=\left(F_{2 n+1}^{(s, 1)}, F_{2 m}^{(s, 1)}, L_{2 n+2 m+1}^{(s, 1)}\right)$ is a solution of $z^{2}-\Delta x^{2}+\Delta y^{2}=\Delta x y z-4$.

Corollary 7. Let $n$ and $m$ be integers, then $(x, y, z)=\left(L_{2 n}^{(s, 1)}, F_{2 m}^{(s, 1)}, F_{2 n+2 m}^{(s, 1)}\right)$ and $(x, y, z)=$ $\left(L_{n}^{(s,-1)}, F_{m}^{(s,-1)}, F_{n+m}^{(s,-1)}\right)$ are solutions of the equation $\Delta z^{2}-x^{2}+\Delta y^{2}=\Delta x y z-4$. Moreover, $(x, y, z)=\left(L_{2 n+1}^{(s, 1)}, F_{2 m}^{(s, 1)}, F_{2 n+2 m+1}^{(s, 1)}\right)$ is a solution of the equation $\Delta z^{2}-x^{2}-\Delta y^{2}=\Delta x y z+4$.

Remark 8. In Corollaries 6 and 7 we obtain the same equations as in [4] for $\Delta=5$. So if $r=1$, then $s= \pm 1$, and if $r=-1$, then $s= \pm 3$. This gives larger families of solutions than in [4].

\section{Acknowledgements}

The author is grateful to the referees for their comments and suggestions which improved the quality of this paper.

\section{References}

[1] Ait-Amrane, L., \& Behloul, D. (2017). On some Diophantine equations involving generalized Fibonacci and Lucas numbers, Colloq. Math., 150(2), 257-268.

[2] Andreescu, T., \& Andrica, D. (2014). Equations with solution in terms of Fibonacci and Lucas sequences, An. Stiint. Univ. "Ovidius” Constanta Ser. Mat., 22 (3), 5-12.

[3] Demirtürk, B. \& Keskin, R. (2009). Integer solutions of some Diophantine equations via Fibonacci and Lucas numbers, J. Integer Seq., 12(8), Article 09.8.7, 14 pp.

[4] Keskin, R. (2014). Three identities concerning Fibonacci and Lucas numbers, Notes on Number Theory and Discrete Mathematics, 20 (5), 44-48. 
[5] Lucas, E. (1878). Theorie des Fonctions Numeriques Simplement Periodiques, Amer. J. Math., 1, 184-240, 289-321.

[6] Ribenboim, P. (1996). The New Book of Prime Number Records, Springer-Verlag, New York.

[7] Ribenboim, P. (2000). My Numbers, My Friends. Popular Lectures on Number Theory. Springer-Verlag, New York. 